\title{
Preparation and Application of Polyurethane Coating Material Based on Epoxycyclohexane-Tetrahydrofuran in Paper Conservation
}

\author{
Juan Liu ${ }^{1}$, Shan-Shan Jin ${ }^{2}$, Ying-Ping $\mathrm{Qi}^{3}$, Yong-Feng Shen ${ }^{3}$ and Hua $\mathrm{Li}^{2, *}$ \\ 1 Department of Pharmacy, Kangda College of Nanjing Medical University, Lianyungang 222000, China; \\ liujuan281@njmu.edu.cn \\ 2 School of Chemical Engineering, Zhengzhou University, Zhengzhou 450001, China; 15737953500@163.com \\ 3 Department of Preservation, Zhengzhou Museum, Zhengzhou 450000, China; k365667769@163.com (Y.-P.Q.); \\ 13215856433@163.com (Y.-F.S.) \\ * Correspondence: lihua@zzu.edu.cn
}

check for

updates

Citation: Liu, J.; Jin, S.-S.; Qi, Y.-P.; Shen, Y.-F.; Li, H. Preparation and Application of Polyurethane Coating Material Based on EpoxycyclohexaneTetrahydrofuran in Paper Conservation. Coatings 2021, 11, 1143. https://doi.org/10.3390/ coatings11091143

Academic Editor: Ioannis

Karapanagiotis

Received: 5 September 2021

Accepted: 18 September 2021

Published: 20 September 2021

Publisher's Note: MDPI stays neutral with regard to jurisdictional claims in published maps and institutional affiliations.

Copyright: (c) 2021 by the authors. Licensee MDPI, Basel, Switzerland. This article is an open access article distributed under the terms and conditions of the Creative Commons Attribution (CC BY) license (https:/ / creativecommons.org/licenses/by/ $4.0 /)$.

\begin{abstract}
Paper cultural heritages are valuable historical records and also abound in cultural resources. Due to its organic property, paper is susceptible to aging, destruction by environmental pollution and human factors. At present, many countries in the world are facing the problem of paper conservation. Coating reinforcement is one of the methods for paper conservation, in which the choice of reinforcing resin is key. A transparent polyurethane, based on epoxycyclohexane (CHO)tetrahydrofuran (THF) copolyether, was adopted in this study. The ring-opening polymerization for generating the $\mathrm{CHO}-\mathrm{THF}$ copolyether took place by the reactants $\mathrm{CHO}$ and THF, in the catalysis of boron trifluoride diethyl etherate, initiation of glycerol. Characterizations of the synthetic copolyether were conducted by infrared (IR) spectroscopy and proton nuclear magnetic resonance $\left({ }^{1} \mathrm{HNMR}\right)$ spectroscopy. The transparent polyurethane was then produced by the CHO-THF copolyether and hexamethylene diisocyanate (HDI) trimer. The influences of different concentrations of polyurethane solution upon the paper tensile strength, elongation, folding endurance, tearing strength, gloss, and brightness were studied. These findings suggest that $10 \%$ polyurethane solution is optimal, not only for greatly improving the paper performance, but also for keeping with the principle of "repair as old". The applied results demonstrate that the polyurethane based on the CHO-THF copolyether has the characteristics of copolyether along with polyurethane, displaying good mechanical properties in paper reinforcement.
\end{abstract}

Keywords: copolyether; polyurethane; paper conservation

\section{Introduction}

Paper cultural heritages such as classics, manuscripts, archives, calligraphies, paintings, and letterheads record human history, culture, science, and technology [1-4]. However, external factors such as environmental pollution, temperature, light, oxygen, water, and microorganisms, and internal factors such as fiber breakage, acidification cause paper aging and performance degradation [5,6]. Accordingly, paper conservation is essential. The coating reinforcement method is effective, with a natural or synthetic resin used as a coating to protect the paper [7]. The broken fibers are reconnected physically or chemically while resin penetrates into the paper, and thus mechanical properties are built up. Furthermore, the coating provides a corrosion inhibition for external factors. Obviously, the choice of reinforcing resin is key. In addition to colorlessness, transparency, and no adverse impact on paper fibers, the resin should also perform an efficient combination with paper fibers. Polyurethane, a synthetic polymer material, has good mechanical properties, weatherability, corrosion resistance, and adhesion [8]. Crosslinking reactions carry out at room temperature between highly reactive isocyanates of polyurethane and hydroxyls of 
paper fibers [9]. Polyurethane and fibers are connected closely through chemical bonds. As a result, paper can be strengthened and mechanical properties can be improved. Main chains of copolyether that contain in general polyurethane are straight, flexible structures lacking rigidity, so it is difficult for polyurethane to possess both excellent mechanical and weathering properties [10-12]. Conversely, main chains of copolyether containing cyclic groups exhibit excellent processing performance, high weathering and hot resistance, and are not easy to crystallize [13]. Polyurethane synthesized from it are less susceptible to crystallize between the soft and hard segment due to the weaker inter-chain forces and lower microphase separation, performing a disordered state with better transparency [14-16]. $\mathrm{CHO}$ has a six-membered alicyclic group with solidity and a ternary epoxy group with high activity. For the ring-opening polymerization, a copolymerization reaction occurs in the polymer system of CHO and THF to produce copolyether. The synthetic copolyether owns both a six-membered alicyclic rigid structure and a flexible butyl structure in the main chain. Based on the proportional control of rigid and flexible segment, rigidity and molecular weight of copolyether are adjustable as required [17]. In addition, as a steric hindrance effect of cyclohexyl, polyurethane is disordered, transparent, and not easily crystallized [17-19]. Polyurethane coating based on CHO-THF presents high gloss and solvent resistance [17].

The CHO-THF copolyether was produced by reactants $\mathrm{CHO}$ and THF, then polyurethane was prepared through the synthetic copolyether and HDI trimer in this study $[18,20]$. The synthetic polyurethane was applied in paper reinforcement. The results demonstrate that paper mechanical properties were improved [21].

\section{Materials and Methods}

2.1. Materials

Materials are illustrated in Table 1.

Table 1. Materials.

\begin{tabular}{ccc}
\hline Material & Specification & Manufacturer \\
\hline CHO & industrial grade & Shenma Group (Pingdingshan, China) \\
\hline THF & analytical grade & Tianjin Komeo Chemical Reagent Co., Ltd. (Tianjin, China) \\
\hline glycerol & analytical grade & Tianjin Komeo Chemical Reagent Co., Ltd. (Tianjin, China) \\
\hline dichloromethane & analytical grade & Tianjin Komeo Chemical Reagent Co., Ltd. (Tianjin, China) \\
\hline boron trifluoride diethyl etherate & analytical grade & $\begin{array}{c}\text { Tianjin Chemical Reagent Plant 3 } \\
\text { (Tianjin, China) }\end{array}$ \\
\hline ammonium carbonate & analytical grade & $\begin{array}{c}\text { Tianjin Chemical Reagent Plant 3 } \\
\text { (Tianjin, China) }\end{array}$ \\
\hline ethyl acetate & analytical grade & Tianjin Komeo Chemical Reagent Co., Ltd. (Tianjin, China) \\
\hline HDI trimer & industrial grade & Bayer (Leverkusen, Germany) \\
\hline dibutyltin dilaurate & analytical grade & Shanghai Aladdin Biochemical Technology Co., Ltd. (Shanghai, China) \\
\hline Xuan paper & - & Xuan Paper Company (Xuancheng, China)
\end{tabular}

\subsection{Synthesis of CHO-THF Copolyether}

The ring-opening polymerization for generating the CHO-THF copolyether took place by the reactants $\mathrm{CHO}$ and THF, in the catalysis of boron trifluoride diethyl etherate, initiation of glycerol $[17,22]$. A total of $20 \mathrm{~mL}$ dichloromethane, glycerol (molar ratio to raw materials being $0.02: 1$ ), boron trifluoride diethyl etherate (molar ratio to raw materials being $0.045: 1$ ), THF (molar ratio to $\mathrm{CHO}$ being 1:1) were added successively into a $250 \mathrm{~mL}$ three-neck flask under the nitrogen protection, stirred for $20-30 \mathrm{~min}$ at $0{ }^{\circ} \mathrm{C}$ for uniform mixing. $\mathrm{CHO}$ was introduced dropwise once every $10 \mathrm{~s}$ in the reaction process, with temperature maintaining at $0{ }^{\circ} \mathrm{C}$ for $6 \mathrm{~h}$. Thereafter, deionized water was given to terminate 
the reaction, as reaction system was adjusted to $\mathrm{pH} 7$ with $10 \%$ ammonium carbonate solution. Transparent copolyether was prepared by water washing, phase separation, and vacuum distillation (oil bath temperature of $110^{\circ} \mathrm{C}$ ) $[8,17,22]$.

\subsection{Synthesis of Polyurethane Based on CHO-THF Copolyether}

With the CHO-THF copolyether, HDI trimer, dibutyltin dilaurate addition to ethyl acetate $\left(6.5 \%, 53 \%, 2 \%, 38.5 \%\right.$ by weight), the reaction proceeded at $70{ }^{\circ} \mathrm{C}$ [20]. Content of isocyanate group (-NCO) was determined every hour. When the content reached $15 \%$, the reaction was stopped [23].

\subsection{Paper Coating}

Polyurethane based on the CHO-THF copolyether was diluted by ethyl acetate. Subsequently, Xuan paper (cut into $210 \mathrm{~mm} \times 297 \mathrm{~mm}$ ) was steeped into different concentrations of polyurethane liquid for $2-5 \mathrm{~min}$. In the end, the treated Xuan paper was dried at room temperature $[18,24,25]$. Coating process is illustrated in Figure 1.

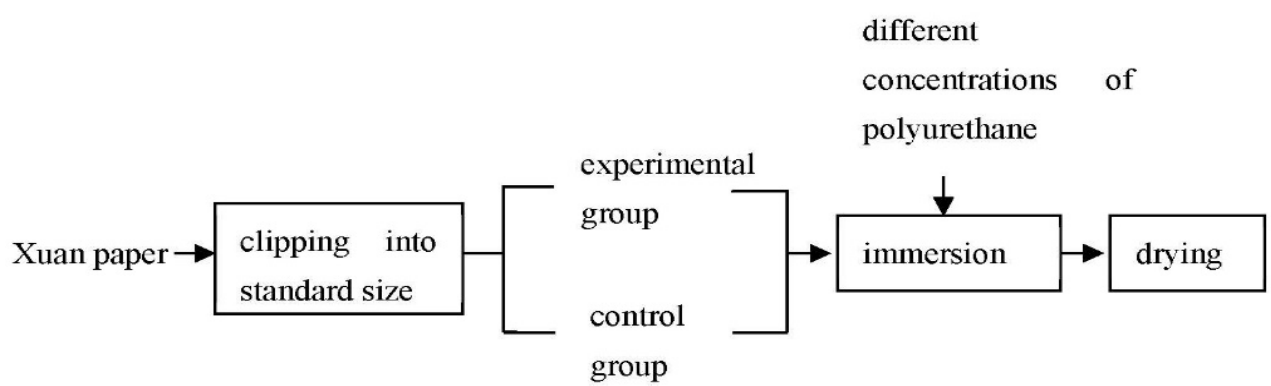

Figure 1. Process of paper coating.

\subsection{Analysis and Testing Methods}

Paper treated by polyurethane was cut into a size of $24.0 \mathrm{~cm} \times 1.5 \mathrm{~cm}$, then 10 strips were taken vertically, the determination of the tensile strength, elongation of the paper were measured at a PN-TT300 (Hangzhou Pin Heng Technology Co. Ltd., Hangzhou, China), with a speed of $20 \mathrm{~mm} / \mathrm{min}$ [26]. Paper was cut into a size of $15.0 \mathrm{~cm} \times 1.5 \mathrm{~cm}$, then 10 strips were taken vertically, determination of the folding endurance was measured at a double-fold instrument PN-NZ135 (Hangzhou Pin Heng Technology Co. Ltd., Hangzhou, China), with a force of $4.9 \mathrm{~N}$ [27]. Paper was cut into size of $6.5 \mathrm{~cm} \times 5.0 \mathrm{~cm}$, then 5 strips were taken vertically, determination of the tearing strength was measured at a PN-TT1000 (Hangzhou Pin Heng Technology Co. Ltd., Hangzhou, China) [28]. Paper gloss was measured at a PN-GM glossmeter (Hangzhou Pin Heng Technology Co. Ltd., Hangzhou, China), with mode chosen as G20, G60, G75 (incident angles are $20^{\circ}, 60^{\circ}, 75^{\circ}$ ) [29], ten tests proceeded at each polyurethane concentration. Paper brightness was measured at a brightness tester of PN-48B (Hangzhou Pin Heng Technology Co. Ltd., Hangzhou, China), with mode chosen as blue, green light diffuse reflectance spectra (R457, Ry) [18,30]; ten tests proceeded at each polyurethane concentration. A FT-IR200 fourier transform infrared spectrometer (Thermo Fisher Scientific, Waltham, MA, USA) was used for IR characterization. An AV II-400 nuclear magnetic resonance spectrometer (Bruker Optics, Karlsruhe, Germany) was applied to determine ${ }^{1}$ HNMR. A NovaNano SEM450 scanning electron microscope was adopted (FEI, Hillsboro, OR, USA).

\section{Results and Discussion}

\subsection{The Reaction Mechanism of CHO-THF Copolyether}

The ring-opening polymerization for generating $\mathrm{CHO}-\mathrm{THF}$ copolyether takes place by the reactants $\mathrm{CHO}$ and $\mathrm{THF}$, in the catalysis of boron trifluoride diethyl etherate, solvent of dichloromethane, initiation of glycerol [22,31]. In the chain initiation stage, an intermediate is generated by the combination of boron trifluoride diethyl etherate and glycerol, which 
then reacts with $\mathrm{CHO}$. The corresponding product is an oxonium ion, possessing a positive charge at the active center. In the chain growth stage, the oxonium ion, $\mathrm{CHO}$, and THF react with each other, resulting in an active product with a carbocation, which makes an atomic attack to the oxygen of monomer, serving as a nucleophilic reaction. In the chain termination stage, $\mathrm{H}_{2} \mathrm{O}$ combines with the active product to terminate the reaction [17]. The reaction mechanism is shown in Scheme 1.

The chain initiation stage:

$$
\left.\mathrm{BF}_{3} \cdot \mathrm{OEt}_{2}+\mathrm{HOCH}_{2} \mathrm{CHOHCH}_{2} \mathrm{OH} \rightleftharpoons\left[\mathrm{BF}_{3} \mathrm{OCH}_{2} \mathrm{CHOHCH}_{2} \mathrm{OH}\right]_{\mathrm{H}}^{\ominus}+\mathrm{BF}_{3} \mathrm{OCH}_{2} \mathrm{CHOHCH}_{2} \mathrm{OH}\right]^{\ominus} \stackrel{\mathrm{OEt}_{2}}{\longrightarrow}
$$

The chain growth stage:

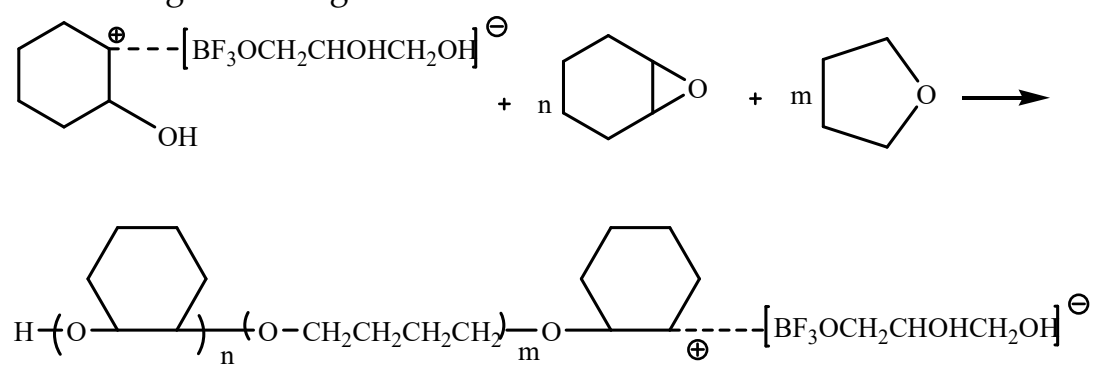

The chain termination stage:

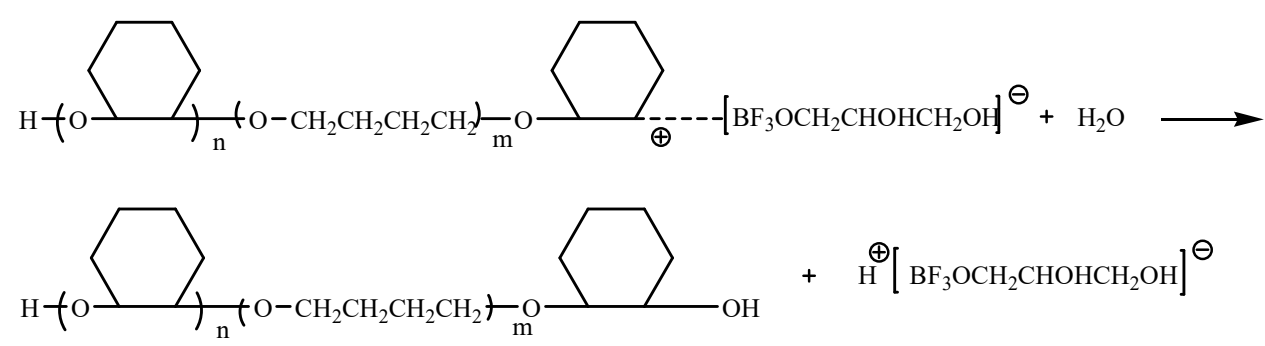

The total reaction:

$(n+1)$<smiles>C1CC[C@@H]2OC2C1</smiles>

$m$<smiles>C1CCOC1</smiles>

$\underset{0^{\circ} \mathrm{C}}{\stackrel{\mathrm{BF}_{3} \cdot \mathrm{OEt}_{2}}{\longrightarrow}}$<smiles>CC1(C)CCCCC1OCCCCCCCO</smiles>

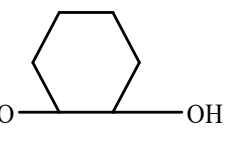

Scheme 1. Reaction mechanism of the CHO-THF copolyether.

The synthesis process of the CHO-THF copolyether is illustrated in Figure 2.

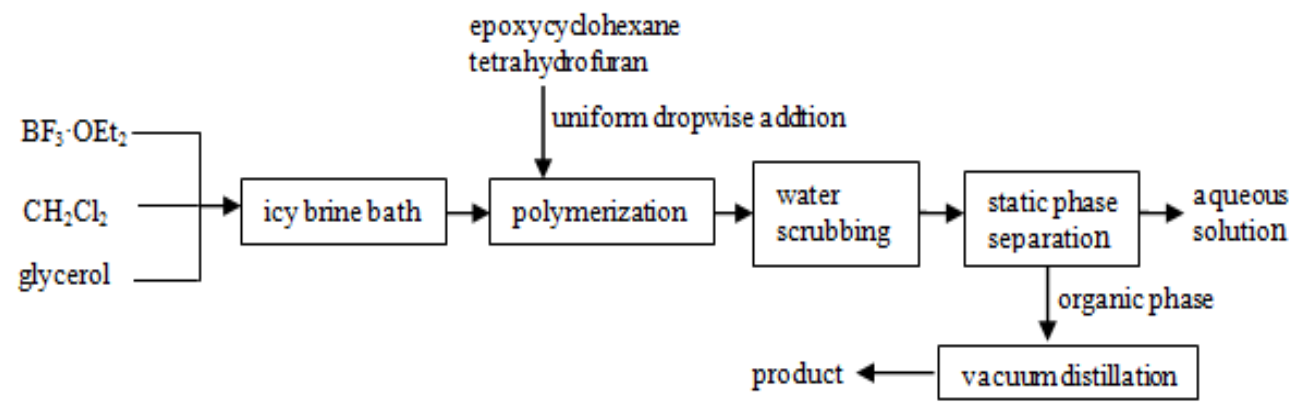

Figure 2. Synthesis process of copolyether. 
As Figure 3 shows, the absorption band at $1088 \mathrm{~cm}^{-1}$ in the IR spectrum is the characteristic absorption peak of aliphatic ether bond, an iconic functional group of the synthetic copolyether. The vibration absorption peak at $3438 \mathrm{~cm}^{-1}$ belongs to the hydroxyl group. The stretching vibration bands of C-H in methylene and methine are at 2929 and $2855 \mathrm{~cm}^{-1}$, respectively. There is also a deformation vibration peak of $\mathrm{C}-\mathrm{H}$ at $1448 \mathrm{~cm}^{-1}$ [17].

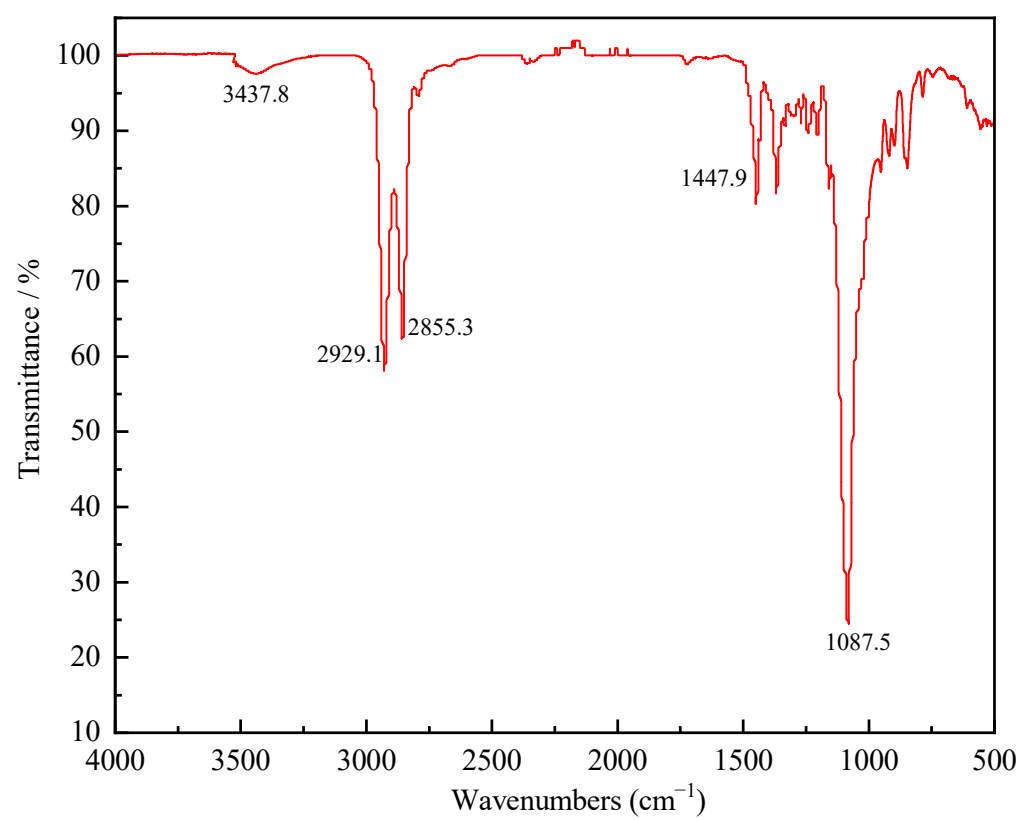

Figure 3. IR spectroscopy of the synthetic copolyether.

${ }^{1} \mathrm{HNMR}$ analysis of the synthetic copolyether is illustrated in Table 2.

Table 2. ${ }^{1} \mathrm{HNMR}$ analysis of the synthetic copolyether.

\begin{tabular}{cc}
\hline Characteristic Functional Groups & Chemical Shift $\boldsymbol{\delta}$ (ppm) \\
\hline$a$ & 3.1 \\
$b$ & 3.5 \\
$c$ & 3.3 \\
$d$ & 1.9 \\
$e$ & 1.6 \\
\hline
\end{tabular}

The representations of $\mathrm{H}$ in $a, b, c, d, e$ are illustrated in Figure 4. Accordingly, the ${ }^{1}$ HNMR spectroscopy of the synthetic copolyether is illustrated in Figure 5.

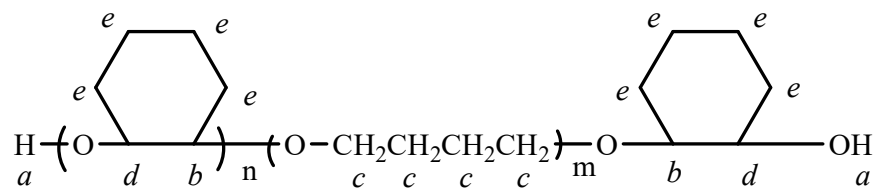

Figure 4. Structure analysis of the synthetic copolyether.

As Figure 5 shows, the proton peak of hydroxyl in $\mathrm{C}-\mathrm{CH}-\mathrm{OH}$ is at $\delta 3.1$, the proton peak of ether in $\mathrm{C}-\mathrm{CH}-\mathrm{O}-\mathrm{C}$ is at $\delta 3.5$, the proton peak of ether in $\mathrm{C}-\mathrm{CH}_{2}-\mathrm{O}-\mathrm{C}$ is at $\delta 3.3$, the proton peak of $\mathrm{C}-\mathrm{CH}-\mathrm{C}-\mathrm{O}$ is at $\delta 1.9$, and the proton peak of $\mathrm{C}-\mathrm{CH}_{2}-\mathrm{C}-\mathrm{C}$ is at $\delta 1.6$. The absorption peaks between $\delta 1.0$ and $\delta 2.0$ belong to the hydrogen of nonadjacent to oxygen in the residual monomers of $\mathrm{CHO}$ and links $\left(-\mathrm{OCH}_{2} \mathrm{CH}_{2} \mathrm{CH}_{2} \mathrm{CH}_{2} \mathrm{O}-\right)$ of THF. The absorption peaks between $\delta 3.0$ and $\delta 4.0$ belong to the hydrogen of adjacent to oxygen in 
the residual monomers. Both the IR and ${ }^{1} \mathrm{HNMR}$ spectrum suggested hydroxyl terminated copolyether was prepared through ring-opening polymerization of $\mathrm{CHO}$ and THF.

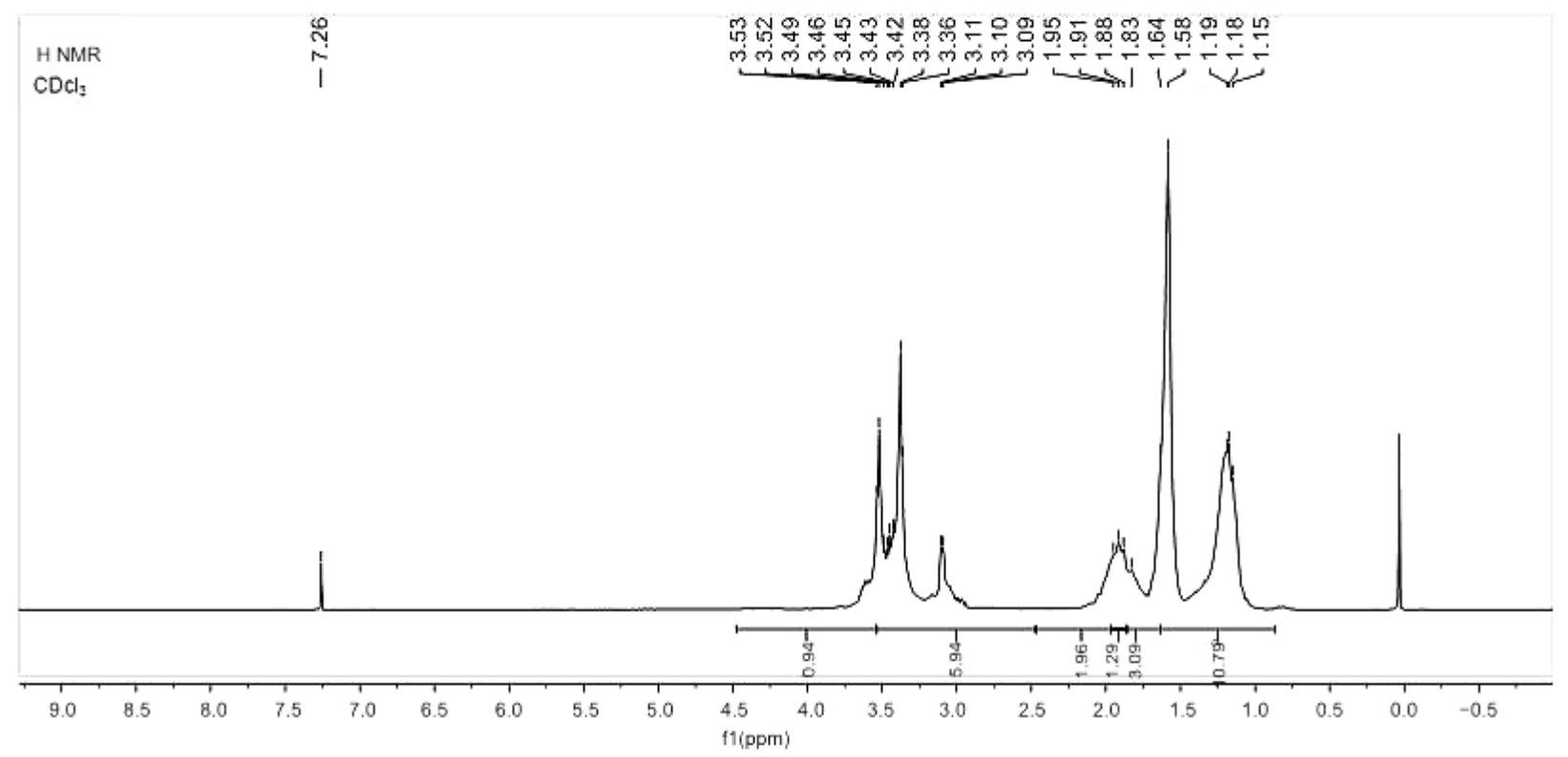

Figure $5 .{ }^{1} \mathrm{HNMR}$ of the synthetic copolyether.

\subsection{Properties of Polyurethane Based on CHO-THF Copolyether}

Polymerization is generated by the reactants CHO-THF copolyether, and HDI trimer. The reaction between high-reactivity isocyanate groups of the HDI trimer and hydroxyl groups of the copolyether is provoked by the catalysis of dibutyltin dilaurate. The reaction mechanism is illustrated in Scheme 2.

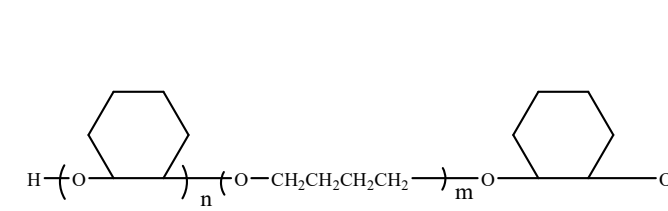

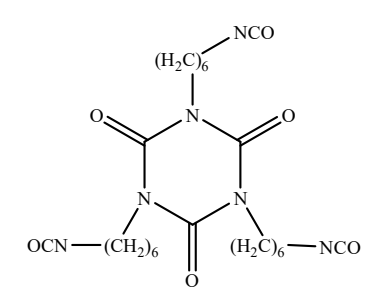

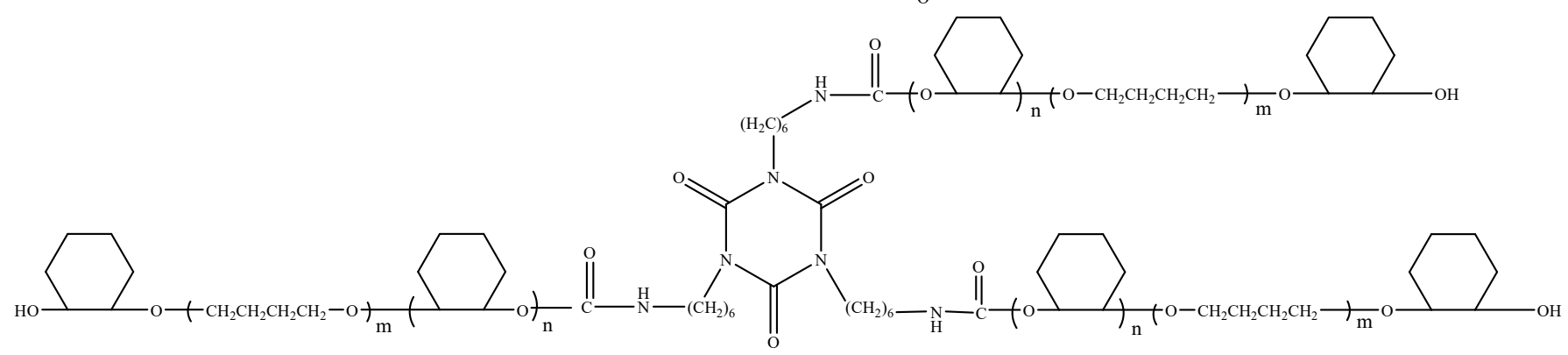

Scheme 2. Reaction mechanism of polyurethane.

\subsection{Application of Polyurethane Based on CHO-THF Copolyether in Paper}

Tensile strength, elongation, folding endurance, and tearing strength of paper were detected as the mass concentration variation of polyurethane [18,19]. As shown in Figure 6a, with increasing mass concentration, tensile strength increased from 1105 to $3616 \mathrm{~N} / \mathrm{m}$, with a growth rate of $227 \%$. Elongation is another key indicator for paper tensile strength. As illustrated in Figure $6 \mathrm{~b}$, with mass concentration rising, elongation reached the peak of $3.45 \%$ at $15 \%$ of mass concentration and then began to descend, with an addition of 
almost $283 \%$. The substitution of hydrogen bonds for carbamate bonds in paper fibers causes a reinforcing bonding action as a three-dimensional crosslinking system forms [9]. Yet the paper may become brittle with the continued growth in mass concentration. As Figure $6 \mathrm{c}$ illustrates, as a sustainable growth of mass concentration, the folding endurance tended to rise and then decline. The folding endurance achieved 1752 times while mass concentration was $10 \%$, with an increase of $866 \%$. Because of the rigid six-membered ring in $\mathrm{CHO}$, the resistant external forces of paper enhance with coating existence. In the folding process, more capabilities of the coated paper fibers are obtained towards holding the original flexibility, which respond to a marked increase in folding endurance and tensile strength simultaneously. On the contrary, the continuous growth of mass concentration makes paper grow thicker, causing polyurethane coating to fracture and become crisper obviously. Thus, the flexibility of the paper declines. As Figure 6d shows, with rising mass concentration, the tearing strength increased firstly, peaking at $185 \mathrm{mN}$ at $15 \%$ of mass concentration, and decreased afterward, with a growth rate of $51 \%$. It also reflects an interaction of the fracture paper fibers with the synthetic polyurethane existence. However, steric hindrance of the polyurethane molecules continues to increase with the sustained growth in mass concentration, and bonding forces of fibers fall to peel the paper.

(a)

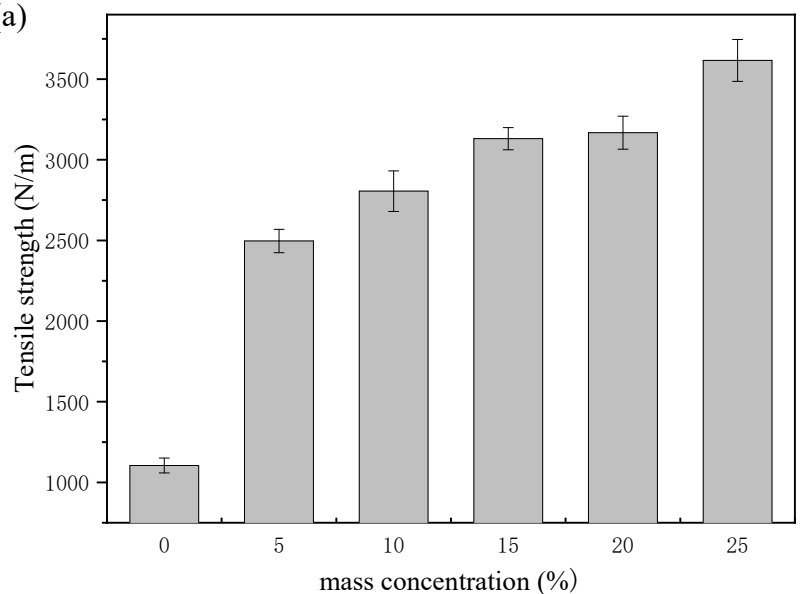

(c)

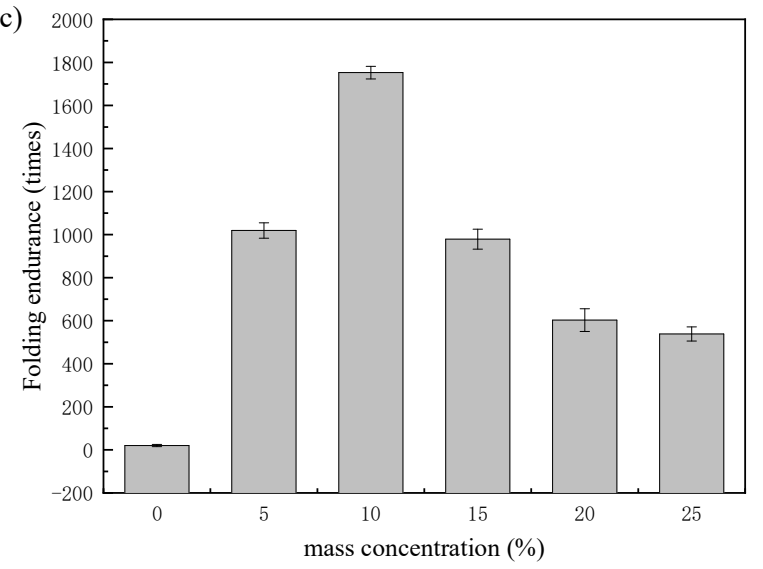

(b)

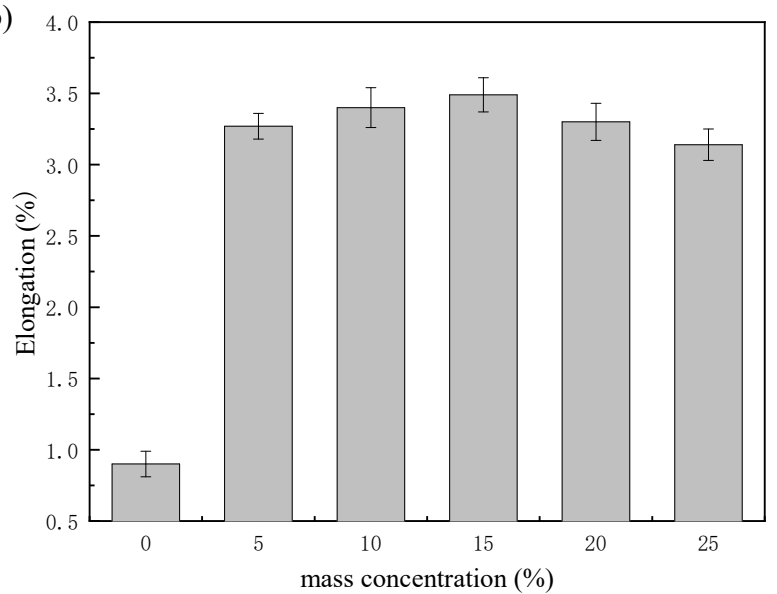

(d)

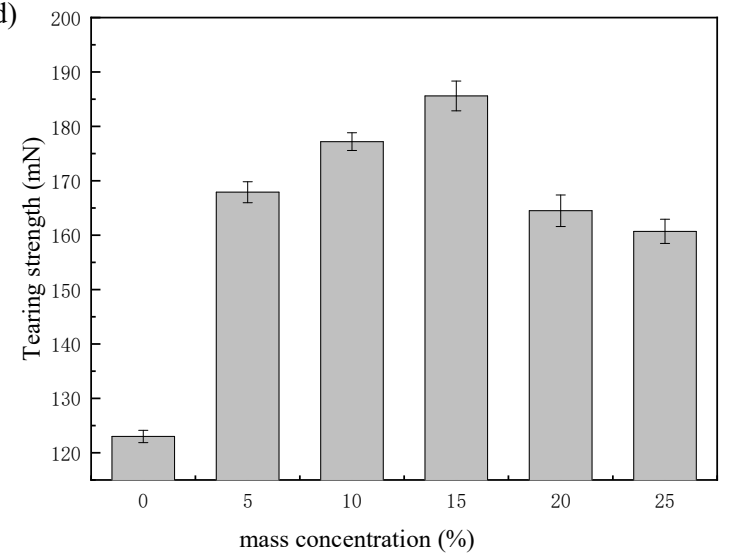

Figure 6. Mechanical properties of the paper treated with different mass concentrations of polyurethane: (a) tensile strength; (b) elongation; (c) folding endurance; (d) tearing strength.

Gloss of the paper was tested as the mass concentration variation of polyurethane, illustrated in Figure 7. 


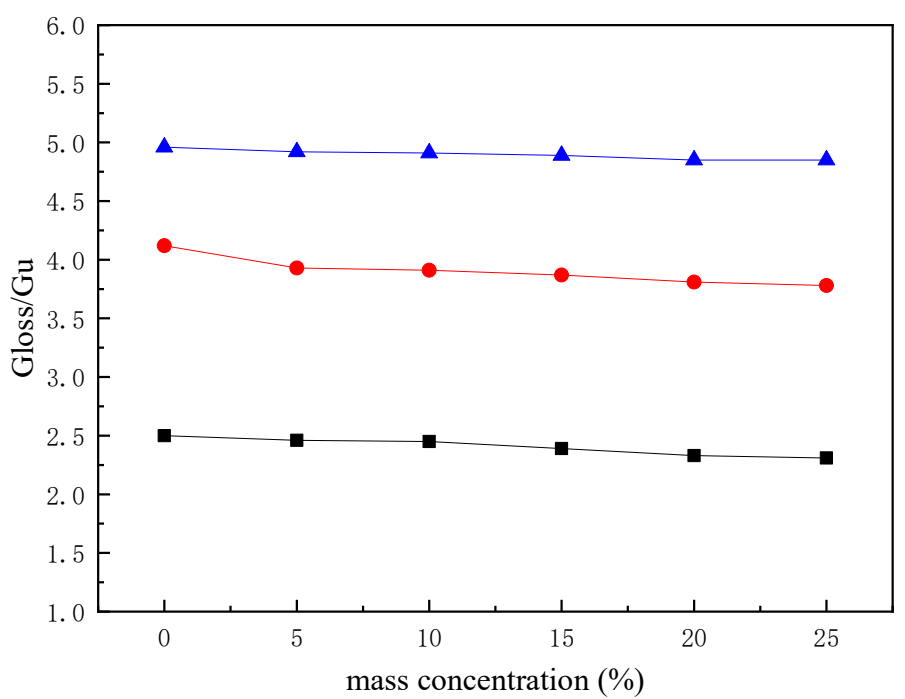

Figure 7. Gloss of the paper treated with different mass concentrations of polyurethane.

G20; • G60; $\Delta$, G75.

As Figure 7 illustrates, with increasing mass concentration, gloss tended to reduce slightly. The gloss of G20, G60, G70 (incident angles are $20^{\circ}, 60^{\circ}, 75^{\circ}$ ) reduced with a decreased percentage of $8 \%, 7.6 \%$, and $2.2 \%$, respectively. The greater the concentration of coated polyurethane when the light hits the reinforced paper, the less reflection occurs, resulting in a reduction in paper's gloss. In general, the gloss affects little by the synthetic polyurethane.

Brightness of the paper was tested as the mass concentration variation of polyurethane, illustrated in Figure 8.

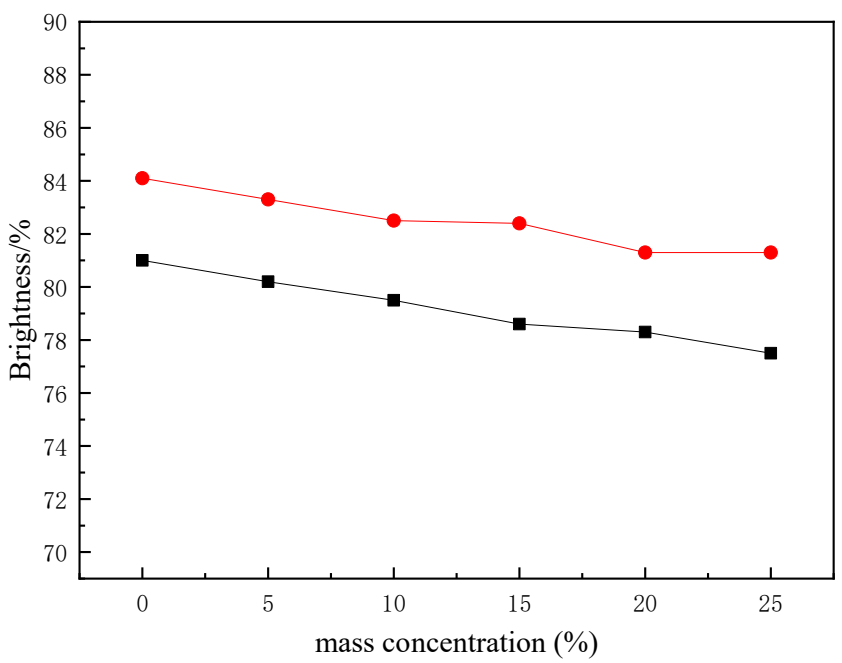

Figure 8. Brightness of the paper treated with different mass concentrations of polyurethane. R457; •, Ry.

As Figure 8 shows, as sustainable growth of mass concentration, the brightness of the paper degraded in a lower degree. R457 represents blue light brightness, reflectivity of blue light exposure. Ry represents green light brightness, reflectivity of green light exposure. The brightness of R457, Ry fell by 4.3\%, 3.3\%. Polyurethane dries to a film on the surface of the paper; as polyurethane concentration increases, there is a certain masking effect on the brightness of the paper. On the whole, the brightness affects little by the synthetic polyurethane. 
For comprehensive appraisal of tensile strength, elongation, folding endurance, tearing strength, brightness, and gloss, the mass concentration of polyurethane based on the CHO-THF copolyether was chosen as $10 \%$.

Paper performance after treatment of polyurethane consolidation solution indicates that gaps in the paper fibers are packed; additionally, bonding effects among fibers are improved, and mechanical properties are enhanced. The synthetic polyurethane has a six-membered alicyclic group with solidity, and the paper anti-reciprocating folding ability at some point is enforced, contributing to the resistant external forces of paper. Meanwhile, in the folding process, more capabilities of the coated paper fibers are obtained towards holding the original flexibility. In summary, tensile strength, folding endurance, elongation, and tearing strength increase due to the polyurethane coating. Polyurethane based on the CHO-THF copolyether owns both a six-membered alicyclic rigid structure and a flexible butyl structure in the main chain. Based on the proportional control of rigid and flexible segments, rigidity and molecular weight of copolyether are adjustable as required. In addition, the steric hindrance effect of cyclohexyl, polyurethane is disordered, transparent, not easily crystallized [17-19]. Polyurethane coating based on CHO-THF presents high gloss and solvent resistance.

Combined with the previous studies, paper performance after treatment of polyurethane based on $\mathrm{CHO}$ homopolyetheryl, $\mathrm{CHO}$-epoxychloropropane $(\mathrm{ECH})$ copolyether, and the $\mathrm{CHO}-\mathrm{THF}$ copolyether were compared and analyzed. The tensile strength of paper in coating increased markedly. For homopolyetheryl-based polyurethane, as mass concentration rose, tensile strength reached the peak of $3039 \mathrm{~N} / \mathrm{m}$ at $20 \%$ of mass concentration, then began to descend, with an addition of almost 175\%. For copolyether-based polyurethane, with increasing mass concentration, tensile strength both tended to increase, and there was a similarity between them. Tensile strength of paper treated by $\mathrm{CHO}-\mathrm{ECH}$-copolyetherbased polyurethane increased from 1105 to $3739 \mathrm{~N} / \mathrm{m}$, with a growth rate of $238 \%$. Tensile strength of paper treated by the CHO-THF-copolyether-based polyurethane increased from 1105 to $3616 \mathrm{~N} / \mathrm{m}$, with a growth rate of $227 \%$. At each mass concentration, the tensile strength of paper in homopolyetheryl-based polyurethane coating was lower than that in copolyether-based polyurethane coating. For elongation, all of them exhibited in homoplastic degree. As sustainable growth of mass concentration, the folding endurance of each tended to rise and then decline. The folding endurance of homopolyetheryl-based polyurethane, $\mathrm{CHO}-\mathrm{ECH}$-copolyether-based polyurethane, and CHO-THF-copolyetherbased polyurethane, achieved discriminatingly in 504, 1801, 1752 times. Particularly, copolyether-based polyurethane was more favorable. As rising in mass concentration, all the tearing strengths raised firstly, peaking at 154,188, $185 \mathrm{mN}$, and decreased afterward. At each mass concentration, the tearing strength of paper in homopolyetheryl-based polyurethane coating was lower than that in copolyether-based polyurethane coating. In addition, the gloss and brightness were affected little by the synthetic polyurethane $[19,25]$. Generally, copolyether-based polyurethane performing in similarity is preferable in the influences of polyurethane solution upon the paper.

The scanning electron microscope (SEM) images of paper are illustrated in Figure 9.

As shown in Figure 9a, the distribution of paper fibers represented as messy and irregular in thickness, and more inter-fiber gaps existed. As Figure 9b illustrates, paper fibers after treatment of polyurethane consolidation solution represented as coarser and were dispersed evenly. It also reflected an interaction of the fracture paper fibers. Furthermore, the inter-fiber gaps were packed by the consolidation solution to make a smoother surface. 

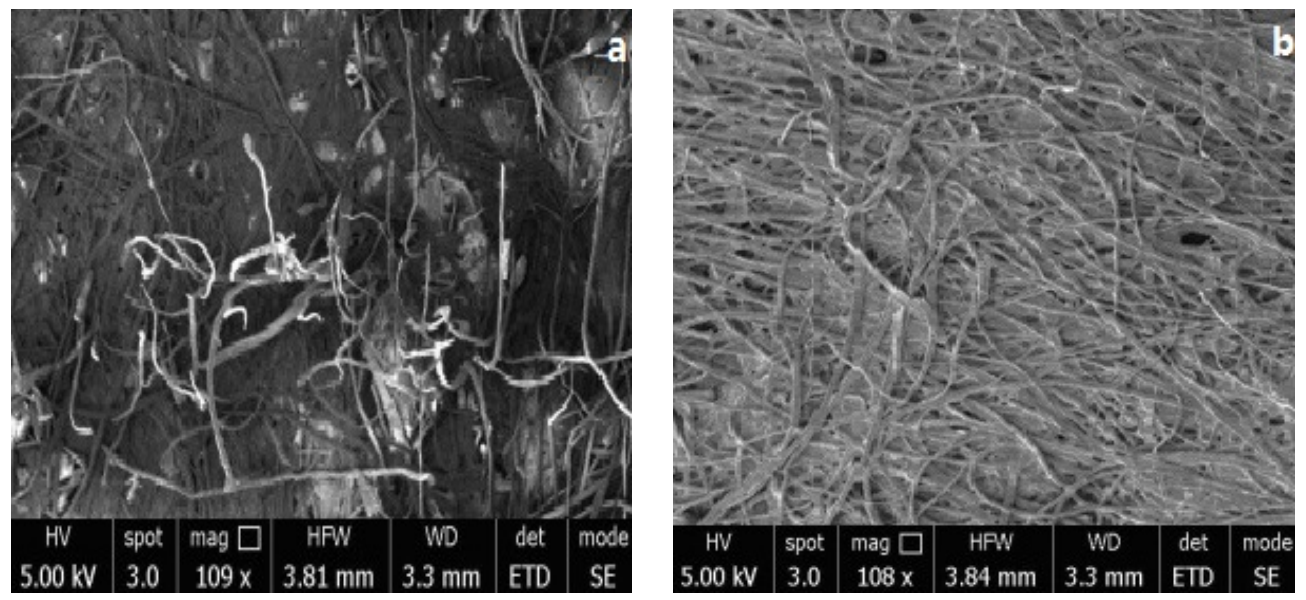

Figure 9. SEM images of Xuan paper treated with polyurethane liquid before (a) and after (b).

\section{Conclusions}

The ring-opening polymerization for generating the CHO-THF copolyether took place by the reactants $\mathrm{CHO}$ and THF, in the catalysis of boron trifluoride diethyl etherate, initiation of glycerol. Characterizations of the synthetic copolyether were conducted by infrared (IR) spectroscopy and proton nuclear magnetic resonance $\left({ }^{1} \mathrm{HNMR}\right)$ spectroscopy. Transparent polyurethane was then produced by the CHO-THF copolyether and HDI trimer. The applied results demonstrate that tensile strength, folding endurance, elongation and tearing strength increase due to the polyurethane coating. Additionally, gloss and brightness displayed a good state when the polyurethane coating forms. Generally, copolyether-based polyurethane performing in similarity is preferable in the influences of polyurethane solution upon the paper. In short, these findings suggest that $10 \%$ polyurethane solution is optimal, not just as greatly improving the paper performance, but as keeping with the principle of "repair as old". The applied results indicate that the polyurethane based on the CHO-THF copolyether has characteristics of copolyether along with polyurethane, exhibiting good mechanical properties in paper reinforcement. The study still needs to be further explored.

Author Contributions: Conceptualization, H.L. and S.-S.J.; methodology, H.L. and S.-S.J.; validation, H.L., J.L. and S.-S.J.; formal analysis, J.L. and S.-S.J.; investigation, H.L. and S.-S.J.; resources, Y.-P.Q. and Y.-F.S.; data curation, S.-S.J., J.L., Y.-P.Q. and Y.-F.S.; literature retrieval and chart making, J.L.; writing-original draft preparation, J.L., S.-S.J. and H.L., revision of manuscripts, J.L., S.-S.J., Y.-P.Q., Y.-F.S. and H.L.; writing-review and editing, H.L.; supervision, H.L. All authors have read and agreed to the published version of the manuscript.

Funding: This research received no external funding.

Institutional Review Board Statement: Not applicable.

Informed Consent Statement: Not applicable.

Data Availability Statement: Not applicable.

Conflicts of Interest: The authors declare no conflict of interest.

Sample Availability: Samples of the compound CHO-THF copolyether are available from the authors.

\section{Abbreviations}

The following abbreviations are used in this manuscript: CHO: epoxycyclohexane, THF: tetrahydrofuran, ECH: epoxychloropropane, HDI: hexamethylene diisocyanate, IR: infrared, ${ }^{1} \mathrm{HNMR}$ : proton nuclear magnetic resonance, SEM: scanning electron microscope. 


\section{References}

1. Afsharpour, M.; Imani, S. Preventive protection of paper works by using nanocomposite coating of zinc oxide. J. Cult. Herit. 2017, 25, 142-148. [CrossRef]

2. Qiao, L.; Chen, K.; Zhao, D.; Zhan, Y.; Min, W.; Huang, Q.; Shen, G.; Zhou, J. The application of poly(methyl methacrylate-co-butyl acrylate-co-styrene) in reinforcing fragile papers: Experiments and computer simulations. Cellulose 2017, 24, 5157-5171. [CrossRef]

3. Camargos, C.H.M.; Figueiredo, J.C.D.; Pereira, F.V. Cellulose nanocrystal-based composite for restoration of lacunae on damaged documents and artworks on paper. J. Cult. Herit. 2017, 23, 170-175. [CrossRef]

4. Jin, S.; Qi, Y.; Shen, Y.; Li, H. A transparent polyurethane based on nanosilica in reinforcing papers. Nord. Pulp Pap. Res. J. 2021, 36, 82-90. [CrossRef]

5. Melo, D.; Sequeira, S.; Lopes, J.; Macedo, M.F. Stains versus colourants produced by fungi colonising paper cultural heritage: A review. J. Cult. Herit. 2019, 35, 161-182. [CrossRef]

6. Wu, X.S. Classification of Plant Fibrous Material with Principal Component Analysis. Trans. China Pulp Pap. $2005,20,10-12$.

7. Xu, J.; Zhang, T.; Jiang, Y.; Chen, Q.; Yang, D.; Qiu, F.; Yu, Z. Synthesis of microcrystalline cellulose/ $\mathrm{TiO}_{2} /$ fluorine/styrene-acrylate coatings and the application for simulated paper cultural relic protection. Cellulose 2020, 27, 6549-6562. [CrossRef]

8. Qiao, Y.H. Synthesis of Polyether Polyol and Preparation of Polyurethane Materials Based on Epoxy Cyclohexane and Epichlorohydrin. Master's Thesis, Zhengzhou University, Zhengzhou, China, April 2018.

9. Jin, S.; Qi, Y.P.; Shen, Y.F.; Li, H. Study on the application of oligomers in paper reinforcement protection. J. Chem. 2018, 1, 229-233.

10. Krol, P. Synthesis methods, chemical structures and phase structures of linear polyurethanes. Properties and applications of linear polyurethanes in polyurethane elastomers, copolymers and ionomers. Prog. Mater. Sci. 2007, 52, 915-1015. [CrossRef]

11. Chen, Q.; Wen, W.-Y.; Qiu, F.-X.; Xu, J.-C.; Yu, H.-Q.; Yang, D.-Y. Preparation and application of modified carboxymethyl cellulose $\mathrm{Si}$ / polyacrylate protective coating material for paper relics. Chem. Pap. 2016, 70, 946-959. [CrossRef]

12. Totolin, M.I.; Neamţu, I. Positive findings for plasma polymer (meth)acrylate thin films in heritage protective applications. J. Cult. Herit. 2011, 12, 392-398. [CrossRef]

13. Yang, B.; Lv, C.L.; Sheng, J.X. High Performance Polymeric Optical Materials; Chemical Industry Press: Beijing, China, 2005.

14. Mu, C.S.; Hunag, K.L.; Li, K.X.; Luo, S.J.; Liu, Y.H.; Huang, S.S.; He, Y.L.; Li, W.G. Study progress in polyether polyol. Chem. Ind. Dev. 2009, 38, 13-18.

15. Qian, B.Z.; Zhu, J.F. Development progress on polyether polyol at home and abroad. Fine Spec. Chem. 2010, 18, 5-12.

16. Adhikari, R.; Gunatillake, P.A.; McCarthy, S.J.; Meijs, G.F. Mixed macrodiol-based siloxane polyurethanes: Effect of the comacrodiol structure on properties and morphology. J. Appl. Polym. 2015, 78, 1071-1082. [CrossRef]

17. Gui, X. Synthesis of CHO-THF Polyether Transparent Polyurethane Materials. Master's Thesis, Zhengzhou University, Zhengzhou, China, November 2017.

18. Jin, S.S. Study on Conservation Materials of Silicon-Based Polyurethane for Paper Relics. Master's Thesis, Zhengzhou University, Zhengzhou, China, August 2019.

19. Liu, J.; Jin, S.-S.; Qi, Y.-P.; Shen, Y.-F.; Li, H. Preparation and Application of Polyurethane Coating Material Based on Epoxy Cyclohexane Protective for Paper. Coatings 2021, 11, 431. [CrossRef]

20. Qi, Y.P.; Shen, Y.F.; Yang, Y.; Li, P.; Li, H.; Jin, S.S. A Reinforcing Fluid for Reinforcing Paper Artefacts. CN Patent CN201810081684.0, 14 August 2018.

21. Jin, S.S. The application of organosilicon modifiedpolyurethane in reinforcing traditional paper. Nord. Pulp Pap. Res. J. 2019, 34, 485-494. [CrossRef]

22. Yang, N.N. Study on Synthesis and Properties of Transparent Polyurethane Materials Based on Epoxy Cyclohexane. Master's Thesis, Zhengzhou University, Zhengzhou, China, September 2016.

23. Yan, L. Deteriorated Paper Conservation with Cellulose Solution Based on NaOH/Urea Solvent. Master's Thesis, Zhengzhou University, Zhengzhou, China, August 2019.

24. Zhang, J.K.; Cao, Y. Determination methods and research progress of NCO in polyurethane. Chem. Adhes. 2016, 38, $294-302$.

25. Liu, J.; Jin, S.-S.; Qi, Y.-P.; Shen, Y.-F.; Li, H. Preparation and Application of Polyurethane Coating Material Based on Epoxycyclohexane-Epoxychloropropane Protective for Paper. Coatings 2021, 11, 1005. [CrossRef]

26. GB/T12914-2008. Paper and Board-Determination of Tensile Properties; Standards Press of China: Beijing, China, 2008.

27. GB/T 457-2008. Paper and Board-Determination of Folding Endurance; Standards Press of China: Beijing, China, 2008.

28. GB/T 455-2002. Paper and Board-Determination of Tearing Resistance; Standards Press of China: Beijing, China, 2002.

29. GB/T 8941-2013. Paper and Board-Measurement of Specular Gloss; Standards Press of China: Beijing, China, 2013.

30. GB/T 7974-2013. Paper, Board and Pulps-Measurement of Diffuse Blue Reflectance Factor-D65 Brightness; Standards Press of China: Beijing, China, 2013.

31. Li, Y.; Li, Z.; Shen, G.; Zhan, Y. Paper conservation with an aqueous NaOH/urea cellulose solution. Cellulose 2019, 26, 4589-4599. [CrossRef] 